\title{
11 First-Principles Atomic-Scale Study of Superlow Friction
}

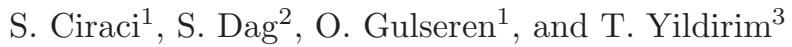 \\ 1 Department of Physics, Bilkent University, Ankara 06800, Turkey \\ ciraci@fen.bilkent.edu.tr \\ 2 Center of Nanophase Materials Science (CNMS) and Computer Science \\ and Mathematics Division, Oak Ridge National Laboratory, 37830, \\ Oak Ridge, TN, USA \\ 3 NIST Center for Neutron Research, Gaithersburg, MA, USA
}

\subsection{Introduction}

Friction is the resistance to the relative motion of two sliding or rolling objects imposed by nonconservative forces [1-3] and, in general, it occurs along with dissipation of mechanical energy and wear. These forces are generated from short- and long-range interactions between the sliding surfaces $[4,5]$. The interaction potential can be either attractive or repulsive depending on the distance between surfaces and also on their relative lateral positions. The moving objects are either in direct contact through asperities, or gaseous, liquid or solid lubricants may be introduced between them to reduce the friction.

The dry sliding friction of two surfaces that are in direct contact through their asperities involves many interesting and complex phenomena, such as adhesion, wetting, atom exchange, the breaking and formation of bonds, as well as elastic and plastic deformation. During the relative motion, phonons are generated and electron-hole pairs are created at the expense of damped mechanical energy. Photons may even be emitted. The nonequilibrium phonon distribution generated locally is dissipated by phonon-phonon and electron-phonon coupling. Simulations of dry sliding friction between a metal asperity and an incommensurate metal surface have revealed unusual atomic processes [6]. For example, the lateral force exhibits a quasiperiodic variation with the displacement of an asperity; each period consists of two different stick-slip processes involving structural transitions. It has also been found that the perpendicular elastic deformation of the substrate that is induced by the sliding object is crucial to the energy damping associated with friction [7]. In certain conditions, due to the elastic deformation of the substrate, the corrugation of the surface potential energy can be inverted under high loading forces. This situation gives rise to the occurrence of a second state (bistability) in the stick-slip motion and anisotropy in the hysteresis curve [7]. It is also very well-known that the stiffer the sliding surfaces, the smaller the friction coefficient [7]. 
The dry sliding friction between atomically flat, commensurate or incommensurate sliding surfaces is perhaps the simplest but most fundamental type of friction in tribology. The relative motion of two commensurate surfaces can take place through repeating stick-slip stages, which can help us to visualize the energy damping under weak elastic deformation. However, the situation is rather complex if the sliding surfaces are incommensurate and undergo an elastic or plastic deformation involving atom exchange and wear. An atomic-scale analysis of the interaction between sliding surfaces is required to understand the nature of nonconservative lateral forces and the various mechanisms of energy damping. In fact, studies based on the Tomlinson's model [8] or calculations made using the Frenkel-Kontorova model [9] have revealed valuable information about the atomic processes involved with friction. Furthermore, the inventions of the atomic force microscope [10] and the friction force microscope $[11,12]$ have had a significant impact on the science of friction and opened up a new field called nanotribology. Nowadays, various atomic processes can be easily observed, and lateral forces in the range of a fraction of nanoNewton $\left(1 \mathrm{nN}=10^{-9} \mathrm{~N}=0.62415 \mathrm{eV} / \AA\right)$ can be measured with precision using these microscopes. As the precision of friction force measurement have increased and various atomic-scale processes have been resolved, atomic-scale simulations involving several atoms have also been performed using realistic empirical potentials [13-18]. Moreover, first-principles studies treating relatively small systems based on density functional theory (DFT) [19] have appeared [20]. First-principles studies have also led to the development of empirical potentials. Theoretical studies, on the other hand, have started to investigate microscopic aspects of energy transfer and energy damping processes [21-24].

Because of the heat generated by the dissipation of mechanical energy and material losses resulting from wear, the objects in relative motion become flawed after some operational time and are eventually destroyed. Since friction results in much resource loss, lowering the friction coefficient has been the principal goal in various fields of science and technology. Lubricants have been used to lower friction coefficients and to eliminate the wear in the machining and transportation industries. Over the last decade, progress made in materials science and surface coating technologies has led to a steady drop in the friction coefficient.

\subsection{Superlow Friction}

\subsubsection{General Theoretical Arguments}

Whether superlubricity - which is somewhat analogous to superconductivity (i. e., a state of matter leading to zero electrical resistivity) or superfluidity (i. e., a liquid state with zero viscosity) - can be achieved has been questioned. The absence of energy damping in mesoscopic objects has been 
pointed out previously [25]. This question can be clarified by examining the energy damping agents involved in friction. These are long- and short-range interactions between two surfaces and various elementary excitations, such as phonons, electron-hole creation, charge density waves, and photon emission. High-energy excitations such as surface plasmons do not contribute to the energy damping process. Bond-breaking or rebonding, atom exchange between surfaces and local surface reconstruction can damp mechanical energy and or mediate excitations. Normally acoustic phonons with small excitation energies can easily be excited and hence contribute to the energy damping process. Experiments performed using a noncontact AFM [26] have shown that the vibration of the tip over the sample gives rise to energy dissipation even if its minimum spacing from the surface is greater than the range of the short-range forces involved [27-30]. This argument eliminates the possibility that an absolutely zero kinetic friction coefficient $\left(\mu_{\mathrm{k}}=0\right)$ can ever occur. Apparently, superfluidity with $\mu_{\mathrm{k}}=0$ cannot be achieved, but a superlow friction coefficient is a target one can reach.

The interaction energy, $E_{\mathrm{i}}(\boldsymbol{\rho}, z)$ between two flat surfaces is a function of their spacing $z$ and their relative lateral positions $\boldsymbol{\rho}=x \boldsymbol{i}+y \boldsymbol{j}$. Usually, the interaction energy is small and attractive (i. e., $\left.E_{\mathrm{i}}<0\right)$ for large $z(z<0)$, but decreases (becomes more attractive) as $z$ decreases. After passing through a minimum, it then starts to increase and eventually becomes repulsive (i. e., $\left.E_{\mathrm{i}}>0\right)$. The attractive interaction energy is specified as an adhesion between the two surfaces and involves the formation of bonds between the surfaces, which may give rise to a high friction coefficient during the sliding motion. Under loading forces, $E_{\mathrm{i}}$ can increase and become repulsive, in which case elastic and or at least local plastic deformations may occur. Substances (solid lubricants, inert gas atoms, etc.) may be placed between the surfaces in order to weaken $E_{\mathrm{i}}$. Under ultrahigh vacuum conditions, friction coefficients as low as $\mu=0.01$ have been observed for $\mathrm{MoS}_{2}$ and diamond-like carbon (DLC) coatings [31-33]. Even if the lubrication of surfaces coated with such low friction coefficient materials appears to be desirable, the low friction coefficient can increase under different ambient and operational conditions. The coating of surfaces with special materials that result in repulsive interactions for a wide range of the loading force $F_{\mathrm{N}}$ is desirable. The loading force will then be balanced by the repulsive force derived from the interaction energy, $F_{z}(\boldsymbol{\rho}, z)=-\partial E_{\mathrm{i}}(\boldsymbol{\rho}, z) / \partial z$, and the atoms on one surface will be prevented from merging into the other surface because a large gap is maintained between the surfaces. In this way, bond-breaking, rebonding and severe deformations can be eliminated. The flights of trains over superconductive rails is reminiscent of the sliding of one coated surface over another when there is a repulsive interaction between them.

In order to reduce the energy damping during the relative motion and hence to lower $\mu_{\mathrm{k}}$, one must also take the force constants (which determine the vibrational frequencies of the atoms) into account. It is well-known that 
the stiffer the sliding surface, the smaller the friction constant. The principal energy-damping agents are phonons, and phonons can be excited by any elastic deformation. Therefore, such elastic deformations are not favored. In this respect, coating materials comprising short and stiff surface bonds are desirable for superlow friction.

\subsubsection{Recent Experimental Progress}

In an effort to lower the friction coefficient, Erdemir et al. [34-36] reported superlow friction and wear between diamond-like carbon (DLC)-coated surfaces using a hydrogen-rich plasma. They achieved kinetic friction coefficients $\mu_{\mathrm{k}}$ as low as 0.001 and wear rates of $10^{-9}$ to $10^{-10} \mathrm{~mm}^{3} / \mathrm{Nm}$ in an inert gas environment under $10 \mathrm{~N}$ load and sliding velocities of $0.2-0.5 \mathrm{~m} / \mathrm{s}$. It has been shown that the magnitude and time-variation of $\mu_{\mathrm{k}}$ are close correlated with the hydrogen content of the source gas. This work by Erdemir and his coworkers was a breakthrough in research into superlow friction and prolonged durability of moving parts in various mechanical applications ranging from the automotive industry to nanotechnology.

\subsection{Theoretical Method}

The structures of sliding surfaces contain several types of defects (such as asperities of different shapes and sizes, vacancies, impurities, domains, etc.). A realistic simulation of dry sliding friction must include all of these defects. Hence, atomistic models of sample surfaces require a large number of atoms. In this respect, the classical molecular dynamics (CMD) method that uses empirical potentials to represent atomic-scale interactions is convenient for simulating friction processes. Recently, various processes have been simulated and new structures have been predicted by using empirical potentials developed for certain systems. Since numerical calculations using these empirical potentials are not time-consuming, large systems comprising several thousands of atoms have been treated. However, the main drawback of CMD simulations appears when a completely new system is treated, particularly when the characteristics of the surface atoms (such as their effective charges and bonds) deviate dramatically from those of the bulk structure. Under these circumstances, whether the empirical potential can be parameterized using the bulk properties becomes questionable. On the other hand, first-principles calculations can provide reliable results for the optimized atomic structure, mechanical, electronic and magnetic properties and phonon density of states of a given system, if it involves a small number (200-300) of atoms. Various mechanisms behind the energy dissipation and estimations of the friction coefficient with upper and lower limits can be elucidated. In this respect, first-principles calculations are superior to classical methods if the system 
can be represented by 200-300 atoms. Besides, first-principles methods are complementary to CDM in that they reveal the correct charge and bond structure and hence aid the development of reliable empirical potentials.

\subsubsection{Details of First-Principles Calculations}

In this study, the atomic processes and forces involved in sliding friction were investigated by carrying out calculations from first principles within DFT. These calculations were proven to yield accurate predictions for many metal and insulator surfaces. Here we present the crucial parameters for the firstprinciples calculations.

The sliding friction is treated either via a supercell method using periodic boundary conditions or by finite-size surfaces using a local basis set. In the supercell method, where the wavefunctions are expressed in momentum space,

$$
\Psi_{n, \boldsymbol{k}}(\boldsymbol{r})=\sum_{\boldsymbol{G}} a_{n, \boldsymbol{k}+\boldsymbol{G}} \exp [-i(\boldsymbol{k}+\boldsymbol{G}) \boldsymbol{r}] .
$$

The magnitude of the largest wave vector sets the cutoff energy, $\hbar^{2} \mid \boldsymbol{k}+$ $\left.G\right|^{2} / 2 m$, and hence the number of plane waves used in the expansion. The ionic potentials are represented by ultrasoft pseudopotentials ([37]; numerical calculations were performed by using the VASP package [38]) and so the cutoff energy is taken to be $300 \mathrm{eV}$. The exchange correlation potential is represented via the generalized gradient approximation [39]. The Brillouin zone corresponding to the supercell is sampled within the Monkhorst-Pack special $\boldsymbol{k}$-point scheme [40].

The sliding surfaces are represented by two infinite slabs made from atomic layers of the coating materials. The atoms in the slabs fall into two different categories, which are treated differently. The first category of atoms, those at the back surfaces of both slabs, are kept fixed in their ideal configurations, $x_{\mathrm{i}}, y_{\mathrm{i}}, z_{\mathrm{i}}$. The layers of fixed atoms represent the sample or coating layers far away from the sliding surface. They are not affected from the friction process. By displacing all of the fixed atoms of one slab relative to the fixed atoms of the other slab, one can achieve a lateral displacement of two slabs and induce a loading force. The atoms at the surface region of the slabs facing each other form the second category, and are relaxed, unlike the atoms from the first category which are fixed at given $x_{\mathrm{i}}, y_{\mathrm{i}}, z_{\mathrm{i}}$ positions. In this way, the processes involved in the relative sliding of the two slabs, including atomic displacements, elastic and plastic deformations, etc., can be modeled from first principles. The atomic positions are optimized by a conjugate gradient method. The lateral components $F_{x}, F_{y}$ and the perpendicular component $F_{z}$ of the net force induced between the two slabs are calculated. We did not include long-range van der Waals forces since they are negligible compared to the perpendicular force $F_{z}$ induced under high loading force $F_{\mathrm{N}}$. 


\subsection{Atomic-Scale Study of Superlow Friction Between Hydrogenated Diamond Surfaces}

\subsubsection{Atomistic Model}

In this section we will present our study of superlow friction between two hydrogenated diamond $(001)-(2 \times 1)$ surfaces performed using a first-principles plane wave method [41]. Hydrogenated DLC (H:DLC) coatings have complex, amorphous structures showing various irregularities. The sliding surfaces cannot be commensurate and they contain irregularly distributed asperities and perhaps voids. We believe that determining the structure of the DLC is itself an important goal, and this issue was addressed previously [42]. However, even if the structure of the H:DLC realized in superlow friction [34-36] as well as the physical and chemical processes associated with friction are stochastic in nature, the local bond orders and the $\mathrm{C}-\mathrm{H}$ bond topology are expected to be similar to various hydrogenated diamond surfaces. Therefore, the interaction between the H:DLC surfaces and the nature of the interaction between these surfaces can be understood by the present model. Clearly, our study does not promise to provide a realistic simulation of the experiment yielding superlow friction [34]. Our objective in this atomic-scale study is to better understand the physical mechanisms involved in the superlow friction observed between hydrogenated DLC-coated surfaces [34]. We hope that the components of the superlow friction revealed in our study will be useful in the development of new coating materials that are stable under the operating conditions desired. In particular, our objective is to develop coating materials that are stable under ambient conditions and to oxidation.

When assessing how simple we can make our model, two features are of particular importance. These are the full relaxation of surface atoms at any instant of the sliding process, and the accurate calculation of the variations in the lateral force components under the constant loading force $F_{\mathrm{N}}$. Diamond(001)- $(2 \times 1)$ surfaces are represented by two slabs facing each other at a specific distance. Each slab consists of six layers of carbon atoms. Carbon atoms at the back surface of each slab are saturated with hydrogen atoms. The atomic structure of each individual slab is first optimized and then the carbon atoms at the sixth layer (at the back surface of the slab) and saturating $\mathrm{H}$ atoms (i. e., those atoms from the first category) are kept at their equilibrium positions. We believe that such a configuration mimics the semi-infinite slab (or a thick coating).

Figure 11.1a illustrates two diamond (001)- $(2 \times 1)$ slabs with H-saturated, fixed back surfaces. The other surfaces of the slabs face each other and are free when the distance $d$ between them is large. The structural parameters of the bare surface, which reconstructs to form dimer bonds, are successfully reproduced. Contour plots calculated for the self-consistent surface charge density are presented in Fig. 11.1b. The dimer and back bonds are clearly seen to have covalent character. 


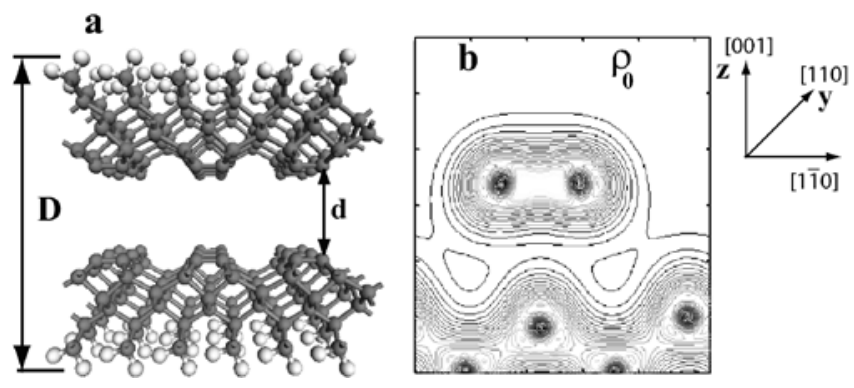

Fig. 11.1. a Two diamond $(001)-(2 \times 1)$ slabs used to model the sliding of two diamond(001) surfaces over each other. Carbon atoms at the back surfaces of the slabs are saturated with $\mathrm{H}$ atoms. The positions of these carbon atoms and those of the saturating $\mathrm{H}$ atoms are fixed at the configuration corresponding to that obtained from the optimization of individual (free) slabs. The distance between the back surfaces of the slabs is $D$, and that between the two sliding surfaces facing each other is $d$. The crystal directions are identified by Cartesian axes shown in the inset. $\mathrm{C}$ and $\mathrm{H}$ atoms are shown by filled and empty spheres, respectively. $\mathbf{b}$ Charge density contour plots for the bare diamond(001)- $(2 \times 1)$ slab for a vertical plane passing through the dimer bond. (Reproduced from [41])

We first calculated the normal force $F_{z}$ which originates from the shortrange interaction between the surfaces of the slabs. To this end, we kept the distance $D$ between the back surfaces of the slabs at each preset value and calculated the total energy of whole system, $E_{\mathrm{T}}(D, \rho)$, and the total force on one of the slabs. Here the total energy and total force are obtained after optimizing the positions of atoms in the second category. We note that, since the two slabs are pressed against each other by fixing $D$, the calculated forces on the atoms at the back surface balance the external (loading) forces which maintain $D$ at a preset value. Therefore, the total calculated vertical force on one of the slabs is equal to the vertical interaction force $F_{z}$. By definition, the loading force $F_{\mathrm{N}}=-F_{z}$. The variation of $F_{z}$ is plotted in Fig. 11.2 with respect to the separation between slab surfaces before relaxation, $d_{o}$, as well as the actual separation, $d$, after the relaxation. The interaction is weak and repulsive for $d>2.75 \AA$, but $F_{z}$ becomes attractive as $D$ decreases and then jumps to contact, attaining a value of approximately $-6 \mathrm{eV} / \AA$. Strong bonds form between the sliding surfaces of two diamond(001)-(2×1) slabs near equilibrium separation corresponding to $F_{z} \simeq 0$. Once a normal force is applied in order to press the slabs against each other, atoms from different surfaces become close to each other at $d \sim 1.5 \AA$ and subsequently $F_{z}$ becomes repulsive. Under these circumstances, since the sliding motion can involve local deformations, bond-breaking and rebonding, the dynamical friction coefficient $\mu_{\mathrm{k}}$ as well as the wear rate are expected to be high. In fact, the dynamical friction coefficient has been measured to be equal to 0.65 for sliding DLC-coated surfaces which are free of hydrogen. 


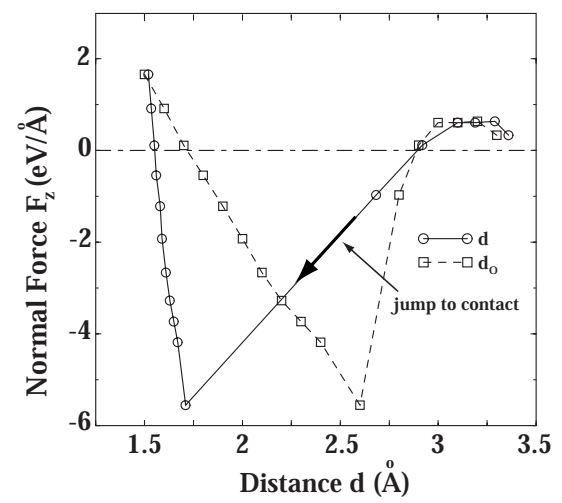

Fig. 11.2. Calculated normal force $F_{z}$ generated when two diamond(001) slabs are pressed towards each other by decreasing $D$ and hence $d$. $d_{o}$ and $d$ correspond to the distance between two sliding diamond(001)- $(2 \times 1)$ surfaces before and after relaxation, respectively.(Reproduced from [41])

\subsubsection{Force Variations in the Sliding Friction of Two Hydrogenated Diamond Surfaces}

First, we will examine the variation in the normal force when the sliding diamond surfaces are hydrogenated. Dangling bonds of carbon atoms on the two slab surfaces facing each other are saturated with $\mathrm{H}$ atoms to form a monohydride phase, i. e., H:diamond(001)- $(2 \times 1)$. Upon the saturation of the surface dangling bonds, the dangling bond surface states disappear and a wide energy gap opens between the valence and conduction bands of the slab. The surface charge density differs dramatically from that of the clean diamond(001)- $(2 \times 1)$. In Fig. 11.3 we show the atomic configurations of the H:diamond $(001)-(2 \times 1)$ surfaces and a contour plot of the surface charge density.

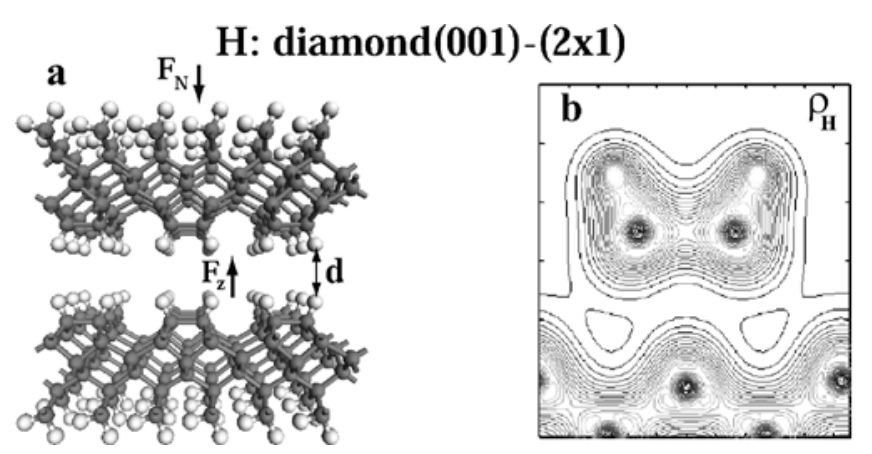

Fig. 11.3. a Atomic configurations of two diamond $(001)-(2 \times 1)$ slabs where the dangling bonds on the surfaces facing each other are saturated with hydrogen atoms to form a monohydride phase $\mathrm{H}$ :diamond(001)- $(2 \times 1)$. $d$ is the spacing between these surfaces, $F_{z}$ the normal force, $F_{\mathrm{N}}$ the loading force. $\mathbf{b}$ Contour plot of total charge density of $\mathrm{H}$ :diamond(001)- $(2 \times 1)$ surface for a vertical plane containing the surface dimer bond. (Reproduced from [41]) 

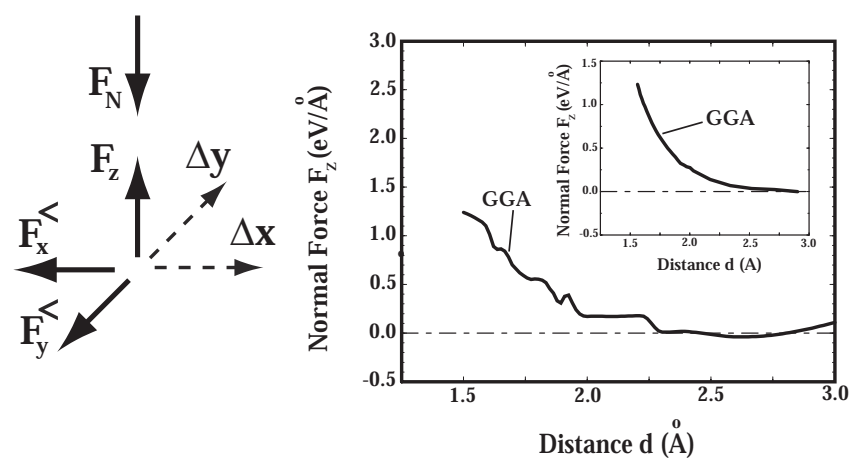

Fig. 11.4. Left: Directions of the loading force $F_{\mathrm{N}}, F_{z}$, lateral force components $F_{x, y}$, and lateral displacements, $\Delta x$ and $\Delta y$. The lateral force component, which acts in the opposite direction to the displacement, is indicated by the superscript " $<$ " symbol. Right: Variation in the calculated normal force $F_{z}$ between the two surfaces of H:diamond(001)- $(2 \times 1)$ as a function of their actual, relaxed separation $d$. $F_{z}$ is generated when two diamond(001) slabs are pressed towards each other by decreasing $D$. The inset shows the variation in the same force between hydrogenated $\mathrm{Si}(001)-(2 \times 1)$ surfaces. (Reproduced from [41])

Moreover, Mulliken analysis indicates that 0.25 electrons are transferred from the $\mathrm{H}$ atom to the $\mathrm{C}$ atom that is bound to it. This situation correlates with the fact that the $\mathrm{C}$ atom is more electronegative than the $\mathrm{H}$ atom. As a result, the $\mathrm{H}$ atom is positively charged. The depletion of electrons on $\mathrm{H}$ atoms induces a repulsive interaction and hence a repulsive $F_{z}$, even for $d<2.5 \AA$, between the H:diamond(001)- $(2 \times 1)$ surfaces. This is the most essential feature for obtaining superlow friction coefficients from H:DLC-coated sliding surfaces. The variation of $F_{z}$ with spacing $d$ is shown in Fig. 11.4. This repulsive force $F_{z}$ keeps the sliding surfaces wide apart at a distance $d$ and balances the loading force $F_{\mathrm{N}}$. As a result, the sliding surfaces are prevented from approaching each other too closely. In this way, $\mathrm{C}-\mathrm{H}$ bond deformation is suppressed to a large extent. It is interesting to note that, like carbon, silicon is also a Group IV element and has a diamond structure. A strong attractive interaction is generated between clean $\mathrm{Si}(001)$ slabs. However, similar to diamond(001) slabs, the attractive interaction turns repulsive upon the hydrogenation of the $\mathrm{Si}(001)$ surfaces, which generates a strong repulsive normal force. It appears that $\mathrm{H}: \mathrm{Si}(001)-(2 \times 1)$ displays features similar to those of $\mathrm{H}$ :diamond(001)- $(2 \times 1)$. We next examine whether this feature, namely the repulsive normal force between surfaces, can lead to a superlow friction coefficient.

\subsubsection{Sliding Friction of Hydrogenated Diamond(001) Slabs}

Having examined the perpendicular variation of $F_{z}$, we now address the following questions. (i) Does the repulsive interaction continue to keep the sur- 
faces wide apart if one of the diamond slabs is laterally displaced relative to the other one? (ii) What is the range of $F_{\mathrm{N}}$ where the repulsive interaction between the surfaces persists without any serious deformation? (iii) Can one obtain an upper limit for the friction coefficient? To answer all of these questions, we carried out a series of first-principles calculations for the interaction energy $E_{\mathrm{i}}$, normal force $F_{z}$, and lateral force $F_{\mathrm{L}}$ corresponding to different loading forces (and hence $D$ ) and displacements $(\Delta x, \Delta y)$ of the upper slab. In these calculations, all of the atoms were relaxed except for the $\mathrm{C}$ and $\mathrm{H}$ atoms at the back surfaces of both slabs. The latter atoms are kept fixed in their ideal configurations after their planes are displaced to different perpendicular and lateral positions by varying $D$ and $(\Delta x, \Delta y)$ in sequential increments. We note that keeping the two back ends of slabs at a specific distance $D$ but relaxing the other atoms induces a loading force $F_{\mathrm{N}}(D)$, which in turn is balanced by $F_{z} . F_{z}$ itself is obtained from the sum of the perpendicular components of the forces calculated for the fixed atoms on one of the slabs, namely $F_{z}=\sum_{i} F_{z, i}$, where $i$ is the index of a fixed atom from one slab. Performing the same sum on the other slab yields $F_{z}$ with the same magnitude but in the opposite direction. Similarly, the lateral forces along the $x$-axis (or the $y$-axis) are obtained from the sum $F_{x(y)}=\sum_{i} F_{i, x(y)}$. Performing ab initio calculations of $E_{\mathrm{i}}, F_{z}, F_{x, y}$ as a function of $d$ (or $D$ ) at different relative lateral positions yields a database of $\Delta x$ and $\Delta y$ values. In these calculations, the values of $D$ were varied in small steps to yield normal forces over an appropriate range of interest.

Figure 11.5 presents the calculated variations in $E_{\mathrm{i}}$ and $F_{z}$ as a function of $d$ for different lateral displacements, $\Delta x$ and $\Delta y$, of the top slab. Here we note that the interaction energy $E_{\mathrm{i}}=E_{\mathrm{T}}-E_{\mathrm{T}, d=\infty}$, where $E_{\mathrm{T}, d=\infty}$ is the total energy corresponding to very large $d$ (or twice the total energy of one slab in the absence of the other one). We note that the variations in $E_{\mathrm{i}}$ and $F_{z}$ are not smooth functions due to the discrete changes in $D$ and to the relaxation of the $\mathrm{C}-\mathrm{H}$ bonds. Note that since $F_{z}$ is always repulsive and strong, even at significantly large spacings, so the sliding surfaces are kept apart, even for large loading forces. As a result, the $\mathrm{C}-\mathrm{H}$ bonds from different surfaces neither merge nor interfere with each other. During the course of sliding, the $\mathrm{C}-\mathrm{H}$ bonds experience neither significant deformation (i. e., bending, stretching or shrinking) nor wear through bonding-rebonding.

If the sliding motion were adiabatic, no energy would be damped during the sliding motion of two commensurate surfaces such as those we treat here. However, this is not the case; various rapid processes generate excitations and give rise to energy damping. However, we will delay a discussion of the microscopic theory of energy damping and dissipation to the next section and instead we now present a global approach to estimating an upper limit for $\mu_{k}$ using the variation in the lateral force obtained from the present calculations. To this end, we consider displacements along the $x$ - and $y$-axes and we derive the variation in lateral force under the given constant loading force using our database, namely $F_{\mathrm{L}=x}\left(\Delta x, \Delta y=0, F_{\mathrm{N}}\right)$ and $F_{\mathrm{L}=y}\left(\Delta x=0, \Delta y, F_{\mathrm{N}}\right)$. 


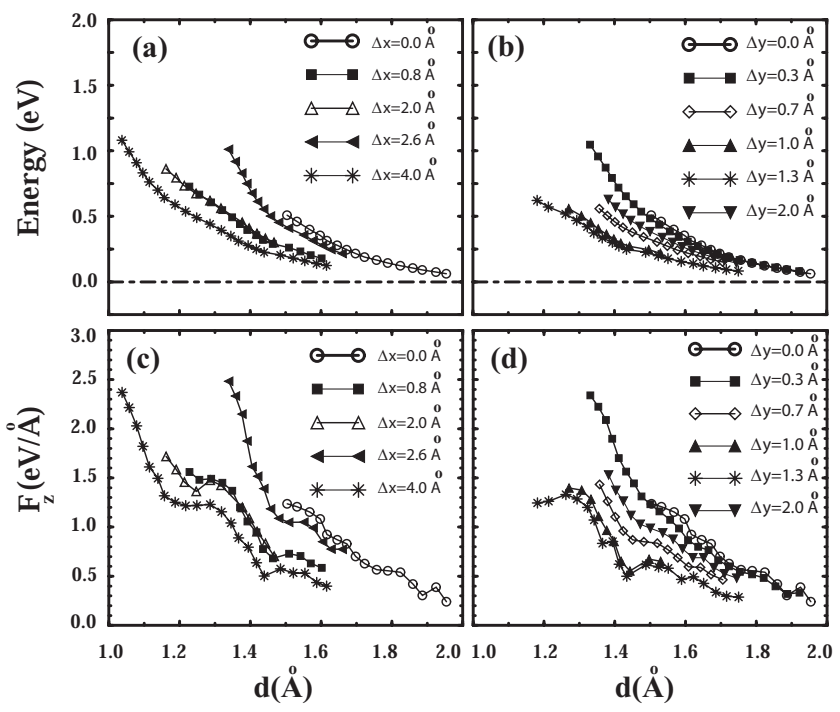

Fig. 11.5. $\mathbf{a}$ and $\mathbf{b}$ Variation in the calculated interaction energy $E_{\mathrm{i}}$ as a function of the perpendicular distance $d$ calculated at different lateral displacements $\Delta x$ and $\Delta y . \mathbf{c}$ and $\mathbf{d}$ The same variation but for the normal force $F_{z}$. Energy and force units correspond to per $(2 \times 1)$ unit cell. (Reproduced from [41])

Keeping the loading force $F_{\mathrm{N}}$ constant is the most difficult part of our study and requires a large number of numerical calculations corresponding to different $\Delta x, \Delta y$ and $D$ values. We considered that the loading force $F_{\mathrm{N}}=1$ and $1.2 \mathrm{eV} / \AA$ per cell, which are actually values that are higher than the loading forces used in the experiment [34] and in practical applications. In this respect, our estimation of $\mu_{k}$ is a stringent test. The variations in $F_{x}$ and $F_{y}$ are illustrated in Fig. 11.6. For the reasons pointed out earlier, the variation in lateral force is not smooth. Since the lateral force is calculated using coarse displacement steps of $D$, the elastic deformations of the slabs and the $\mathrm{C}-\mathrm{H}$ bonds induced by sliding are released suddenly. This gives rise to the stick-slip process described by Tomlinson's model [8]. Of course, there are error bars involved in the calculation of forces. In particular, achieving the constraint of a constant loading force via the limited number of data points in the database generated through ab initio calculations can lead to hysteric variations in the lateral force. Now, as an ad hoc approach to estimating $\mu_{k}$ in an energy-damping medium, we assume that the work done by the lateral force $F_{\mathrm{L}}$ (i. e., when it is parallel to the direction of motion as denoted by $F_{\mathrm{L}}^{>}$) is totally lost. Then the average friction force is calculated by $\bar{F}_{\mathrm{f}}=\int F_{x}^{<} \mathrm{d} x / R$, where $R$ is the period of the motion.

Using the data in Fig. 11.6, we can extract the average friction force, $\bar{F}_{\mathrm{f}} \sim 0.05 \mathrm{eV} / \AA$ for $F_{\mathrm{N}}=1 \mathrm{eV} / \AA$ and $\bar{F}_{\mathrm{f}} \sim 0.07 \mathrm{eV} / \AA$ for $F_{\mathrm{N}}=1.2 \mathrm{eV} / \AA$. Then the kinetic friction coefficient is calculated from $\mu_{\mathrm{k}}=\bar{F}_{\mathrm{f}} / F_{\mathrm{N}}$ to be ap- 

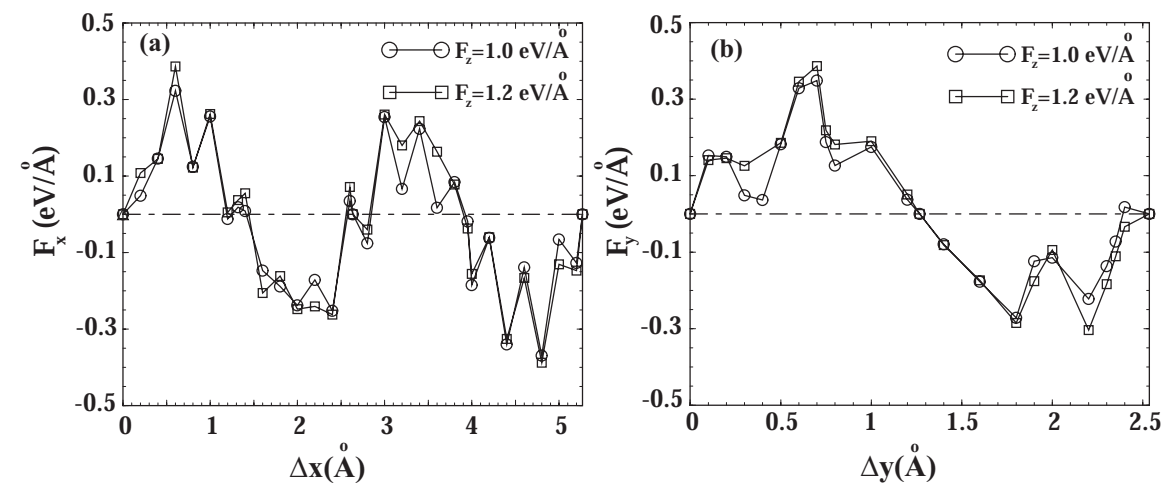

Fig. 11.6. a Variation in the lateral force, $F_{\mathrm{L}=x}[\mathrm{in} \mathrm{eV} / \AA$ per $(2 \times 1)$ cell $]$ as a function of the displacement $\Delta x$ of the top slab relative to the bottom one. $\mathbf{b}$ Same as a but for the displacement $\Delta y$. During the course of sliding, the loading force $F_{\mathrm{N}}$ is taken to be approximately constant. (Reproduced from [41])

proximately 0.05 in both cases. A more realistic estimation could be obtained from $\bar{F}_{\mathrm{f}}=\int\left(F_{x}^{<}+F_{x}^{>}\right) \mathrm{d} x / R$ if the lateral force variation was calculated precisely. Although the force variations shown in Fig. 11.6 are too crude to obtain precise values, $\mu_{\mathrm{k}}$ has been calculated for the sake of comparison to be $\sim 0.01$.

It should be noted that during the sliding of commensurate surfaces the lateral forces acting on each atom or cell are added constructively to yield a high total lateral force. These lateral forces are, however, conservative, and do not give rise to energy damping if the sliding motion is adiabatic. In the case of incommensurate surfaces, the total lateral force is lower due to the cancellations. H:DLC-coated surfaces can be viewed to be incommensurate except that the disorder gives rise to higher energy damping. Consequently, the above estimation of $\mu_{\mathrm{k}}$, obtained from hydrogenated diamond surfaces with the assumption that all mechanical energy stored into elastic energy is damped, is an upper limit for H:DLC-coated surfaces; however, it is still too low.

\subsubsection{Microscopic Theory of Energy Damping}

The mechanisms of energy damping and energy transfer from sliding objects or lubricants have been studied theoretically and experimentally [14, 20-22, 43-45]. In the sliding friction of commensurate surfaces, the elastic energy (which is related to the interaction energy $E_{\mathrm{i}}$ ) $V_{\mathrm{T}}$ exhibits a periodical variation with the displacement; the period is determined by the lateral lattice parameters of the sliding surfaces. In the present case, $V_{\mathrm{T}}$ attains its maximum value when the dimer bonds of the two surfaces face each other, but it becomes minimum when the upper slab is displaced by half of the unit cell. During sliding, $V_{\mathrm{T}}$ varies between maximum and minimum values. If 
the variation is adiabatic there will be no energy damping; mechanical energy is stored into elastic energy mainly through the deformation of $\mathrm{C}-\mathrm{H}$ bonds, and the first half of the period will be released as kinetic energy during the second half of the period. As for the lateral force with components $F_{x, y}=-\partial V_{\mathrm{T}}(\boldsymbol{r}) / \partial x, y$ it will have also the same period as $V_{\mathrm{T}}$. Moreover, it is conservative for fully adiabatic sliding motion. First, it is parallel to the direction of sliding, then it becomes antiparallel so that $\int F_{\mathrm{L}} \mathrm{d} \eta=0$ for fullperiod displacement along the direction of the vector $\boldsymbol{\eta}=x \boldsymbol{i}+y \boldsymbol{j}$. However, the sliding motion is not adiabatic but instead involves sudden changes which can create various types of excitations, in particular nonequilibrium phonons, at the expense of the mechanical energy of the sliding objects.

In the sliding friction of hydrogen-saturated diamond(001)-(2×) surfaces and also $\mathrm{H}$ :DLC, the characteristics of the $\mathrm{C}-\mathrm{H}$ bonds are crucial to the damping of mechanical energy. The $\mathrm{C}-\mathrm{H}$ bonds are associated with a salient surface phonon [46] stretch mode of $\hbar \Omega_{q} \sim 360 \mathrm{meV}$ and a bending mode of $150 \mathrm{meV}$. The $\mathrm{C}-\mathrm{H}$ bonds are short and stiff and cannot be easily excited to large amplitude vibrations in order to dissipate mechanical energy.

Excitation of phonons over the thermal equilibrium distribution with ambient temperature $T_{o}$ is the prime mechanism in energy damping. It involves two stages. (i) Excitation of nonequilibrium phonon distribution. (ii) Dissipation of excess phonons from the sample. Both processes are sample specific; in other words they depend on the sample materials, the atomic structures of the sliding surfaces and operational conditions. In particular, one needs to know the phonon frequency spectrum (or the density of states $D(\Omega)$ ). In principle, the density of phonon frequencies can be calculated if the atomic structures and force constants of the sliding objects are known. If the sliding takes places over a number of asperities, the situation becomes even more complex. In what follows we present a concise theory of a phononic energy damping process through a single asperity.

Let us consider a mode frequency $\Omega_{q}$ and denote the occupation numbers of the corresponding phonon for the ambient temperature $T_{o}$ and for high temperature $T$ as $n_{q}^{o}$ and $n_{q}$, respectively. Here, $n_{q}$ denotes Planck's distribution for a given $\Omega_{q}$ and $T$. We take $T>T_{o}$. Therefore, the excess phonons for this particular mode are expressed as

$$
\Delta n_{q}=n\left(\Omega_{q}, T\right)-n^{o}\left(\Omega_{q}, T_{o}\right) .
$$

Here $q$ is the mode index, including the polarization. The equilibrium state of the sample (or asperity) can be expressed by the occupation number representation as $\Psi\left(n_{1}^{o}, n_{2}^{o}, \ldots n_{q}^{o}, \ldots n_{3 N}^{o}\right)$, with $3 N$ being the degrees of freedom in the motion of $N$ atoms of the system. Subsequent to a deformation, the occupation number state becomes $\Psi\left(n_{1}, n_{2}, \ldots n_{q}, \ldots n_{3 N}\right)$. Here, the crucial problem is to relate the excess phonon density, $\Delta n_{q}$, to the deformation. In principle, a given deformation state in terms of the displacements of individual atoms, $u_{i, x}, u_{i, y}$ and $u_{i, z}$, can be expressed in normal coordinates by 
using the appropriate transformation. In other words, this problem reduces to finding the vibrational states of a system (consisting of $N$ atoms connected by springs) when its preset deformation is suddenly released. In order to provide a fundamental understanding of the phononic dissipation, one can follow a simpler approach and relate the deformation $u_{q}$ to the number of excited phonons by using a semiclassical equation,

$$
\delta V_{q}=\sum_{q} M \Omega_{q}^{2} u_{q}^{2} / 2 .
$$

Here, $\delta V_{q}$ is the mechanical energy used to excite $\Delta n_{q}$ excess phonons with frequency $\Omega_{q}$, namely $\delta V_{q}=\hbar \Omega_{q} \Delta n_{q}$. We note that the amount of energy damped by phonons upon the release of one of the deformed states is the sum of the phonon excitation energies over the mode index $q, V_{\mathrm{T}}=\sum_{q} \delta V_{q}$. Knowing $n_{q}=\Delta n_{q}+n_{q}^{o}$, and using Planck's distribution, one can estimate the local temperature $T$ corresponding to the excited phonons.

Having calculated the excited phonons subsequent to the release of one deformed state, we now discuss the dissipation of excess phonons. The process is closely related to the transfer of energy through molecules and has been treated in several theoretical and experimental studies. The decay of $n_{q}$ to $n_{q}^{o}$ is usually expressed by a rate equation,

$$
n_{q}(t)=n_{q}^{o}+n_{q}(t=0) \exp \left[-R\left(\Omega_{q}\right)\right] .
$$

Here $R\left(\Omega_{q}\right)$ is sample-specific and obtained from scaling arguments. Model calculations on a $\mathrm{Cu}$ asperity consisting of 14 atoms by Buldum et al. [22] showed that low-energy modes experience the highest excitation probabilities but the lowest decay rates. Therefore, low-energy modes determine the phononic energy damping. Moreover, calculations based on nonequilibrium statistical mechanics and Keldysh Green's function formalism [24] show that the excess phonon distribution dissipates within a picosecond if the couplings to the substrate are strong.

\subsubsection{Effect of Oxidation}

The most serious issue is that the superlow friction coefficient obtained from H:DLC-coated surfaces cannot be sustained under ambient conditions [34,35]. The oxygen atom could potentially destroy the superlow friction when the $\mathrm{H}$ :DLC coating is exposed to the air. In what follows, we clarify the effect of oxygen on the hydrogenated DLC coating leading to superlow friction. To test the effect of oxygen, we placed $\mathrm{O}$ atoms at different sites on the H:diamond(001)- $(2 \times 1)$ surface. Upon relaxation, the system attains the minimum energy configuration, whereby $\mathrm{O}$ atoms break surface bonds to form new $\mathrm{C}-\mathrm{O}-\mathrm{C}$ or $\mathrm{C}-\mathrm{O}-\mathrm{H}$ and $\mathrm{C}-\mathrm{O}$ bonds, and hence they become attached to the surface. Fortunately, they attack the $\mathrm{C}-\mathrm{H}$ bonds to form $\mathrm{C}-\mathrm{O}-\mathrm{H}$ radicals. Charge transferred to $\mathrm{O}$ from $\mathrm{H}$ and $\mathrm{C}$ makes the $\mathrm{O}$ atom negatively 


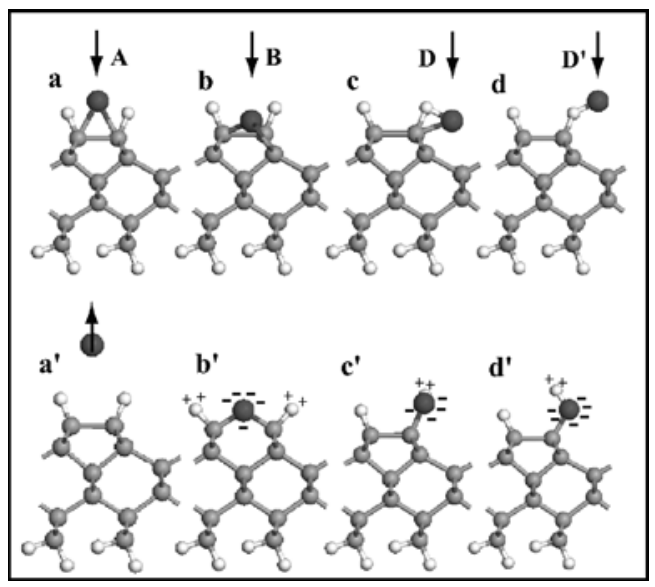

Fig. 11.7. Calculated atomic configurations showing the effect of an oxygen atom on the H:diamond(001)- $(2 \times 1)$ surface. $\mathbf{a}-\mathbf{d}$ The oxygen atom is placed at different sites in the surface unit cell before the relaxation of the surface. a'-d' Atomic structure and bonding after relaxation of the system. The adsorbed oxygen atom is negatively charged. (Reproduced from [41])

charged, as shown in Fig. 11.7. In this way, the interaction between two atoms on different surfaces can be attractive when they carry charges of different polarities. As a result, the steady and strong repulsive interaction between the H:diamond(001)- $(2 \times 1)$ surfaces gradually becomes weaker or turns attractive. Eventually, the superlow friction ends.

\subsection{Conclusions}

This work reports on an extensive study of the interaction between two bare and hydrogenated diamond(001)- $(2 \times 1)$ surfaces. The interaction between bare surfaces is strongly attractive up to a small distance between surfaces $d \sim 1.5 \AA$, at which point it becomes repulsive. Hydrogen atoms donate charge to the carbon atom and become positively charged. This appears to be the most important ingredient of the superlow friction. The repulsive interaction persists at any relative position of the sliding surfaces, and is strong even at large distances from each other, preventing $\mathrm{C}-\mathrm{H}$ bonds from merging. Strong and stiff $\mathrm{C}-\mathrm{H}$ bonds and the stiff diamond crystal prevent large amounts of energy from being dissipated. It was found that oxygenation of surfaces under atmospheric conditions destroys the steady repulsive interaction.

Acknowledgement. This work was supported by Scientific and Technological Council of Turkey, TÜBÍTAK under Grant No. TBAG-104T537.

\section{References}

1. F.P. Bowden and D. Tabor, Friction and Lubrication (Methuen, London, 1965).

2. E. Rabinowitz, Friction and Wear (Wiley, New York, 1965). 
3. B.N.J. Persson, Sliding Friction: Physical Principles and Applications (Springer-Verlag, Berlin, 2000).

4. J.N. Israelachvili, Intermolecular and Surface Forces (Academic Press, London, 1985).

5. S. Ciraci, A. Baratoff and I.P. Batra, Phys. Rev. B 42, 7168 (1990); S. Ciraci,

E. Tekman, A. Baratoff and I.P. Batra, Phys. Rev. B 46, 10411 (1992).

6. A. Buldum and S. Ciraci, Phys. Rev. B 55, 12892 (1997).

7. A. Buldum and S. Ciraci, Phys. Rev. B 55, 2606 (1997).

8. G.A. Tomlinson, Philos. Mag. 7, 905 (1929).

9. J. Frenkel and T. Kontorova, Phys. Z. Sowjet, 13, 1 (1938).

10. G. Binnig, C.F. Quate and Ch. Gerber, Phys. Rev. Lett. 56, 960 (1986).

11. C. M. Mate, G.M. McClelland, R. Erlandsson and S. Chiang, Phys. Rev. Lett. 59, 1942 (1987).

12. E. Meyer, R. Overney et al., Phys. Rev. Lett. 69, 1777 (1992)

13. A. P. Sutton, JB. Pethica, J. Phys.: Condens. Matter 2, 5317 (1990).

14. M. Cieplak, E. D. Smith and M. O. Robins, Science 265, 1209 (1994).

15. B. Bhushan, J.N. Israelachivili and U. Landman, Nature, 347, 607 (1995).

16. M. R. Sorensen, K. W. Jacobsen and P. Stoltze, Phys. Rev. B 53, 2101 (1996).

17. A. Buldum and S. Ciraci, Phys. Rev. B 57, 2468 (1998).

18. A. Buldum and S. Ciraci, Phys. Rev. B 60, 1982 (1999).

19. P. Hohenberg and W. Kohn, Phys. Rev. B 136, B864 (1964); W. Kohn and L.J. Sham, Phys. Rev. 140, A1133 (1965).

20. W. Zhong and D. Tomanek, Phys. Rev. Lett 64, 3054 (1990); D. Tomanek, W. Zhong and H. Thomas, Europhys. Lett. 15, 887 (1991).

21. A. Buldum, D.M. Leitner and S. Ciraci, Europhys. Lett. 47, 208 (1999).

22. A. Buldum, D.M. Leitner and S. Ciraci, Phys. Rev. B 59, 16042 (1999).

23. A. Ozpineci, D. M. Leitner and S. Ciraci, Phys. Rev. B 62, 10558 (2000).

24. A. Ozpineci and S. Ciraci, Phys. Rev. B 63, 125415 (2001).

25. J.B. Sokoloff, Phys. Lett. 71, 3450 (1993).

26. F.J. Giessibl, Science 267, 68 (1995).

27. M. Bammerlin, R. Lüthi, E. Meyer, A. Baratoff, J. Lu, M. Guggisberg, Ch. Gerber, L. Howald, and H.-J. Güntherodt, Probe Microsc. 1, 3 (1997).

28. M. Gauthier and M. Tsukada, Phys. Rev. B 60, 11716 (1999).

29. M. Guggisberg, M. Bammerlin, R. Lüthi, Ch. Loppacher, F. Battiston, J. Lu, A. Baratoff, E. Meyer and H.-J. Güntherodt, Appl. Phys. A 66, S245 (1998).

30. L.N. Kantorovich, Phys. Rev. B 64, 245409 (2001).

31. J.M. Martin, C. Donnet Th. Le Mogne, and Th. Epicier, Phys. Rev. B 48, 10583 (1993).

32. I. Sugimoto and S. Miyake, Appl. Phys. Lett. 56, 1868 (1990).

33. C. Donnet and A. Grill, Surf. Coat. Technol. 94-95, 456 (1997).

34. A. Erdemir, O.L. Eryilmaz and G. Fenske, J. Vac. Sci. Technol. A 18, 1987 (2000).

35. A. Erdemir, Surf. Coat. Technol. 146-147, 292 (2001).

36. J.A. Heimberg, K.J. Wahl and I.L. Singer and A. Erdemir, Appl. Phys. Lett. 78, 2449 (2001).

37. D. Vanderbilt, Phys. Rev. B 41, 7892 (1990).

38. Numerical calculations were performed by using the VASP package: G. Kresse and J. Hafner, Phys. Rev. B 47, 558 (1993); G. Kress and J. Furthmüller, ibid 54, 11169 (1996). 
39. J.P. Perdew, J.A. Chevary, S.H. Vosko, K.A. Jackso, M.R. Pederson, D.J. Singh and C. Fiolhais, Phys. Rev. B 46, 6671 (1992).

40. H.J. Monkhorst and J.D. Pack, Phys. Rev. B 13, 5188 (1976).

41. S. Dag and S. Ciraci, Phys. Rev. B 70, 241401 (2004).

42. G.T. Gao, P.T. Mikulski, and J.A. Harrison, J. Am. Chem. Soc. 124, 7202 (2002); G.T. Gao, P.T. Mikulski, G.M. Chateauneuf and J.A. Harrison, J. Phys. Chem. B 107, 11082 (2003).

43. L.S. Levitov, Europhys. Lett. 8, 449 (1989).

44. B.N.J. Persson, Phys. Rev. B 44, 3227 (1991); B.N.J. Persson and A.I. Volotkin, J. Chem. Phys. 103, 8679 (1995).

45. A. Dayo, W. Alnasrallah, and J. Krim, Phys. Rev. Lett. 80, 1690 (1998).

46. B.D. Thoms and J.E. Butler, Phys. Rev. B 50, 17450 (2000). 